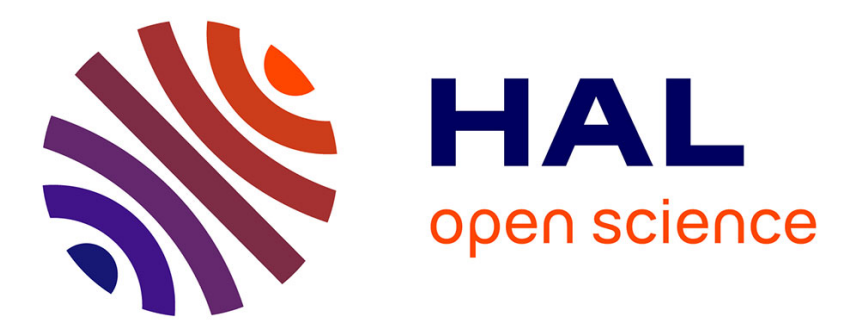

\title{
Influence of particles on the transition to turbulence in pipe flow
}

\author{
Jean-Philippe Matas, Jeffrey F. Morris, Elisabeth Guazzelli
}

\section{To cite this version:}

Jean-Philippe Matas, Jeffrey F. Morris, Elisabeth Guazzelli. Influence of particles on the transition to turbulence in pipe flow. Philosophical Transactions of the Royal Society of London. Series A, Mathematical and Physical Sciences (1934-1990), 2003, 10.1098/rsta.2003.1173 . hal-00252134

\section{HAL Id: hal-00252134 \\ https://hal.science/hal-00252134}

Submitted on 18 Feb 2020

HAL is a multi-disciplinary open access archive for the deposit and dissemination of scientific research documents, whether they are published or not. The documents may come from teaching and research institutions in France or abroad, or from public or private research centers.
L'archive ouverte pluridisciplinaire HAL, est destinée au dépôt et à la diffusion de documents scientifiques de niveau recherche, publiés ou non, émanant des établissements d'enseignement et de recherche français ou étrangers, des laboratoires publics ou privés. 


\title{
Influence of particles on the transition to turbulence in pipe flow
}

\author{
By JeAn-Philippe Matas, JeFFrey F. MorRis $\dagger$ \\ A N D ÉLISABETH GUAZZELLI \\ Institut Universitaire des Systémes Thermiques Industriels, CNRS-UMR 6595, \\ Technopôle Château Gombert, 13453 Marseille Cedex 13, France
}

We investigate by experiment the influence of suspended solids upon the transition to turbulence in pipe flow. The particles are monodisperse and neutrally buoyant with the liquid. The role of the particles on the transition depends upon both the volume fraction, $\phi$, and particle size. Below a critical particle diameter, particles alter the transition to larger critical Reynolds numbers for all $\phi$. In contrast to this, larger particles move the transition to smaller Reynolds numbers for small $\phi$, but they delay the transition at larger concentration.

Keywords: particle; turbulence; suspension; intermittency

\section{Introduction}

The stability of tube flow as the flow rate, and hence the Reynolds number, increases is a classic problem dating from the work of Reynolds (1883), yet it is still not completely solved. In the laminar regime, the flow is described by a parabolic profile known as Poiseuille flow. Above a critical Reynolds number, the laminar flow becomes unstable. Turbulent regions grow and are convected in the pipe; this is the intermittent regime. When the flow rate is further increased, the flow becomes fully turbulent (Tritton 1988).

In fact, in tube flow, the transition to turbulence cannot be simply described by a single critical Reynolds number. The situation is more complex and the transition happens to be globally subcritical: the flow is linearly stable for all flow rates and a finite amplitude instability is needed to trigger the transition (Drazin \& Reid 1981). The transition threshold depends on the amplitude of the perturbation applied to the flow. Experiments have been performed where laminar motion was observed as far as $R e \approx 10^{5}$, but it has also been observed that the transition in pure fluid can occur as soon as $R e \approx 2100$, provided that the perturbation is strong enough to allow the growth of turbulent 'puffs' (Tritton 1988). Recent studies have investigated with different kinds of perturbations the nature of the unstable modes, either in the inlet region or in the fully developed flow (see, for example, Eliahou et al. 1998; Darbyshire \& Mullin 1995).

$†$ Present address: Halliburton Energy Services, 2600 S. 2nd Street, Duncan, OK 73536, USA. 
overflowing reservoir

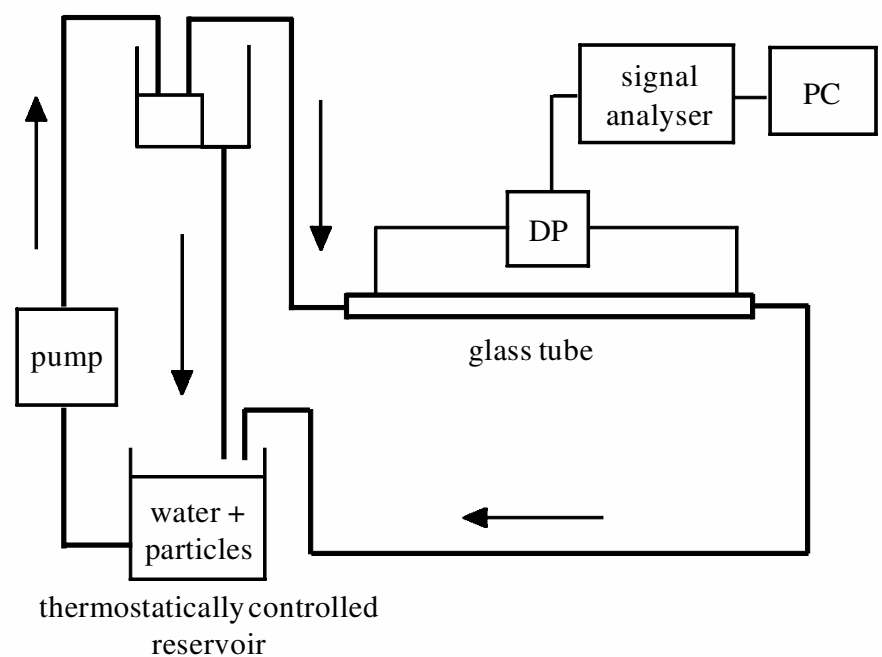

Figure 1. Sketch of the experimental apparatus.

There exists a significant body of literature on the influence of particles on turbulence. Much of the work in this area of study is directed towards understanding how the particle size, in dimensional form or in relation to the viscous length-scale of the fluid, affects the turbulence intensity or the distribution of the particles at a bulk or local scale (see, for example, Li et al. 1999). A review by Crowe et al. (1996) addresses much of the literature in this area up to its date of publication. The noted work considers fully developed turbulence, and we emphasize that here we address a quite separate, although potentially related, question. The objective of the present work is to understand how transition to turbulence is impacted on by the presence of suspended solids in the simplest case of neutral buoyancy. More specifically, we focus upon determining the point of transition between the laminar and the intermittent regime as a function of the particle volume fraction, $\phi$, of the suspension. Because the particles are neutrally buoyant and largely drag free, the present work is related to recent work (Bottin et al. 1997, 1998) that has examined the global subcritical stability behaviour of planar Couette flow, $u_{x} \sim y$, forced by the presence of a single spherical bead or a spanwise wire fixed at $y=0$. We note, however, that the present work also has a practical aspect, as it is related to pipeline flow of slurries.

\section{Experimental set-up}

The experimental apparatus test section was a horizontal $2.6 \mathrm{~m}$ long glass tube with inner diameter $D=14 \mathrm{~mm}$. The tube was thus longer than the entry length $L_{\mathrm{e}} \sim 1 \mathrm{~m}$ necessary for the laminar flow to fully develop at $R e \approx 2000$. In order to ensure that flow in the pipe was undisturbed by perturbations from a pump, the flow was driven by gravity. The suspension was delivered to the tube by overflow from a tank positioned at a fixed height to an outlet of variable height, passing through the glass tube as indicated in figure 1. A Moineau progressing cavity pump (PCM model MR2.6H24) carried the suspension back to the overflowing tank, but was isolated from the flow through the glass tube. 


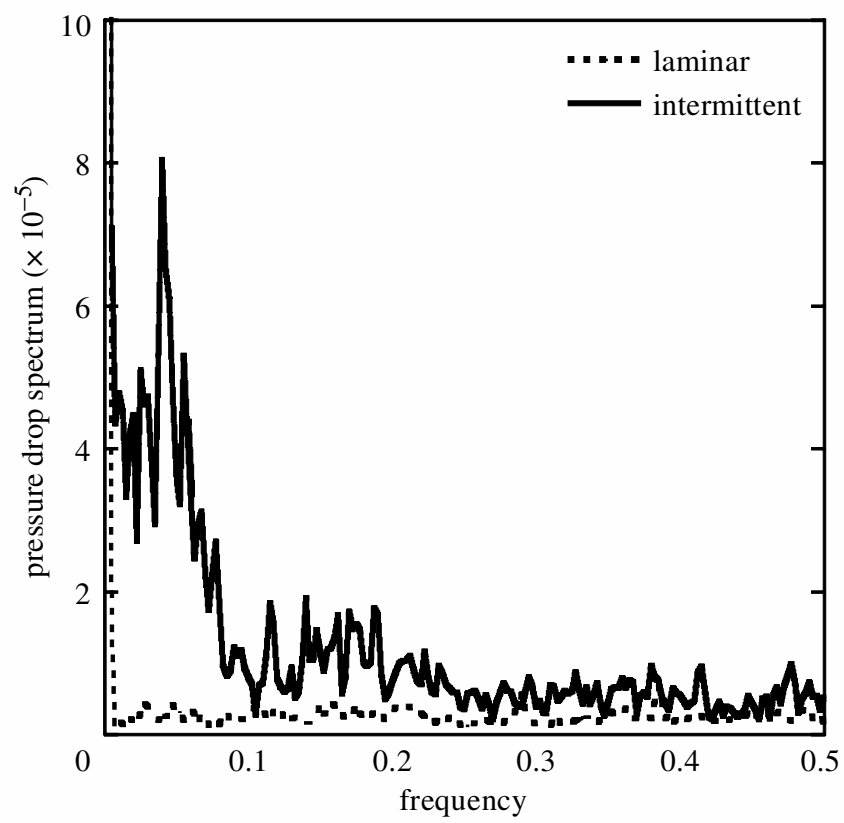

Figure 2. Fluctuations of the pressure drop across the pipe for the laminar and the intermittent regime in a suspension of particle volume fraction $\phi=0.1$. The zero frequency value of the spectrum, related to the mean value of the pressure drop, is much larger and is not represented on the graph.

With these flow conditions, the transition for pure fluid took place at $R e \approx 2300$, but the flow was very sensitive to any kind of perturbation applied to the pipe. In order to control the transition with a known perturbation, a ring (solid annulus) of thickness $1.5 \mathrm{~mm}$, fitting tightly into the tube, was inserted in the pipe at its entrance. The perturbation produced by the ring then controlled the transition from laminar flow and brought the transition to $R e \approx 2100$, which is the lowest value that could be reached with this kind of perturbation. The influence of the particles on the transition was studied using this ring.

Four sizes of spherical polystyrene particles, with diameters $d=40 \pm 6 \mu \mathrm{m}$ (from Kodak, Rochester, NY, USA), $215 \pm 25 \mu \mathrm{m}, 510 \pm 60 \mu \mathrm{m}$ and $780 \pm 110 \mu \mathrm{m}$ (all from Maxiblast, South Bend, IN, USA) and density $\rho=1.051 \pm 0.001 \mathrm{~g} \mathrm{~cm}^{-3}$ were used in the experiment. To obtain neutral buoyancy, the densities of the fluid and of the particles were matched. We chose as the fluid a mixture of $22 \%$ glycerol and $78 \%$ water by mass at $25{ }^{\circ} \mathrm{C}$. The fluid was maintained at this temperature by a thermostatically controlled reservoir in the flow loop. The viscosity of the fluid mixture at this temperature is $\mu=1.64 \pm 0.04 \mathrm{cP}$.

A classical way to detect the pipe-flow transition for pure fluid is to inject dye and to observe the wavering of the streak as it passes down the pipe (Tritton 1988). In the present experiment with particles, direct visualization of the particle motion in the suspension is a very straightforward way of observing the birth and convection of 'turbulent puffs' in the flow. Whereas motion in the laminar regime is characterized by parallel particle trajectories, motion in a turbulent puff presents a strong mixing in the radial direction. When the flow rate is increased, we observe more and more 
turbulent puffs to appear in the flow, and finally these merge once the turbulent regime is established.

To provide a more quantitative indicator of transition, we also measure the pressure drop between the entrance and the end of the glass tube with electronic manometers (Newport Omega PX 154). Pressure fluctuations due to the larger pressure drop caused by the turbulent puffs can be clearly identified with a signal analyser (Hewlett Packard 3562A). Figure 2 shows pressure-drop spectra in the low-frequency range for the laminar flow and at the onset of intermittency, both for a particle volume fraction $\phi=0.1$. The spectrum in the intermittent regime displays strong fluctuations for frequencies under $0.1 \mathrm{~Hz}$. Using this spectral signature, the onset of intermittency can thus be clearly identified. In order to check that this spectrum was not related to the pump, we observed the pressure fluctuations in the flow driven by the pump: the pressure spectrum was then observed to consist of 'continuous noise' of the type shown by figure 2, plus a strong peak at the frequency of the pump rotation with its associated harmonics. By contrast, the fluctuations when the flow is driven by gravity are observed to have only the 'continuous noise'. Furthermore, this noise was determined to be unchanged when the pump was off and the overflow tank was filled manually. Consequently, the spectrum observed in the gravity-driven flow is considered to be truly independent of the pump. We could not identify any clear frequency in this pressure spectrum, beyond the fact that fluctuations associated with intermittence are of frequencies smaller than $0.1 \mathrm{~Hz}$. The order of magnitude of this frequency could suggest that it is related to the structure of intermittent flow in the pipe, characterized by an alternation of laminar and turbulent regions: this limiting value may thus be given by the ratio of the mean flow velocity $U_{\mathrm{m}}=0.2 \mathrm{~m} \mathrm{~s}^{-1}$ to the length of the pipe (1.6 m beyond the entry length) in the conditions of the experiment $(R e \sim 2100): f_{1}=U_{\mathrm{m}} / L=0.13 \mathrm{~Hz}$. However, no clear variation of the pressure signal was observed for transition at larger flow rates, where the frequency $f_{1}$ is significantly increased.

The determination of transition was made by determining the midpoint between the larger flow rate, where laminar flow is observed (a flat spectrum for the pressure fluctuations and visualization of a quiet flow), and the smaller flow rate, where the flow is 'strongly intermittent' (the spectrum of pressure fluctuations is like the intermittent one in figure 2, and there is at the same time an alternation of quiet and strongly turbulent regions in the pipe). This method allowed very good reproducibility of the measurements. It was used to determine the threshold of transition for all the measurements, except for the large volume fraction measurements $(\phi>0.25)$ and the high flow rate situations $(R e>4500)$, where the pressure drop across the pipe exceeds the range of the pressure gauges. In the latter cases, the threshold was only determined by visualization.

Measurements of the critical Reynolds number associated with the start of intermittency were carried out for each particle size, at concentrations $0 \leqslant \phi \leqslant 0.30$. When the threshold of intermittency was reached, we measured the critical flow rate, $Q_{\mathrm{c}}$, and deduced the critical Reynolds number, $R e_{\mathrm{c}}=4 Q_{\mathrm{c}} \rho / \pi \mu D$, with the length-scale the pipe diameter, $D$. To measure $Q_{\mathrm{c}}$ and $\phi$, we collected a given volume of the suspension without altering the flow, by capturing fluid at the outlet to the 'thermostatically controlled reservoir' in figure 1 . The time needed to get this volume yielded $Q_{\mathrm{c}}$. The particles collected in this volume were then sieved, rinsed, dried and weighed to obtain the mean particle volume fraction $\phi$ in the suspension flow. 


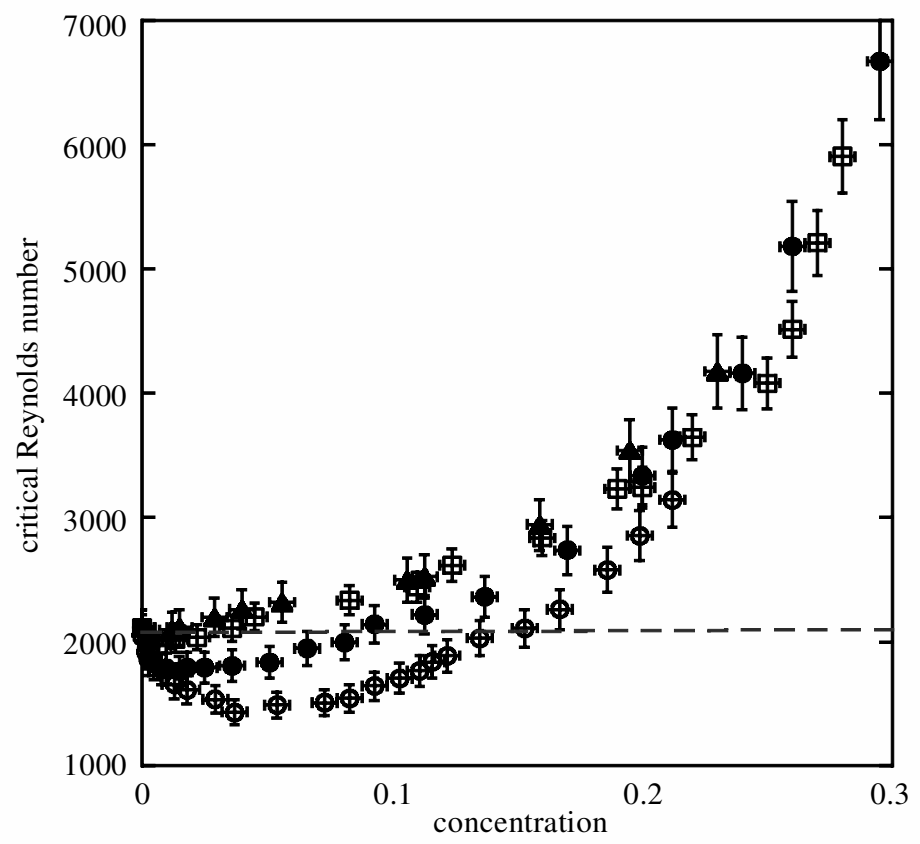

Figure 3. Critical Reynolds number, $R e_{\mathrm{c}}$, as a function of the volume fraction of the suspension for the different sets of particles. The error bars correspond to the uncertainties in the identification of the intermittent regime and in the measurement of the viscosity. $\mathbf{\Delta}, d=40 \mu \mathrm{m}$; $\square, d=215 \mu \mathrm{m} ; \bullet, d=510 \mu \mathrm{m} ; \circ, d=780 \mu \mathrm{m}$.

\section{Results}

Figure 3 shows the critical Reynolds number as a function of the particle volume fraction for the different sets of particles. The results indicate two different situations.

(i) For small particles with $D / d \geqslant 70$, such as the 40 and $215 \mu \mathrm{m}$ particles, the transition is moved to larger Reynolds numbers. $R e_{\mathrm{c}}$ is a monotonically increasing function of the particle volume fraction. Furthermore, the data for the two sizes collapse on a single curve, so that, at a given $\phi$, the threshold of transition does not depend on the size of the particles in this range of $D / d$.

(ii) For large particles with $D / d \leqslant 70$, such as the 510 and $780 \mu \mathrm{m}$ particles, the behaviour depends on the concentration. For small concentrations the transition is moved to lower Reynolds number. The magnitude of the effect, meaning the difference in transition $R e$ from its value at $\phi=0$, depends on the size of the beads. Conversely, for large concentrations $(\phi>0.2)$ the transition is delayed to larger $R e$ and the curve for the $510 \mu \mathrm{m}$ particles tends to collapse on the curves found for the smaller particles.

\section{Discussion}

The behaviour for small particles seems simpler, since it is independent of the size of the particles. The observed delay to the transition, when the Reynolds number is expressed in terms of the pure liquid viscosity, is expected, based upon an 


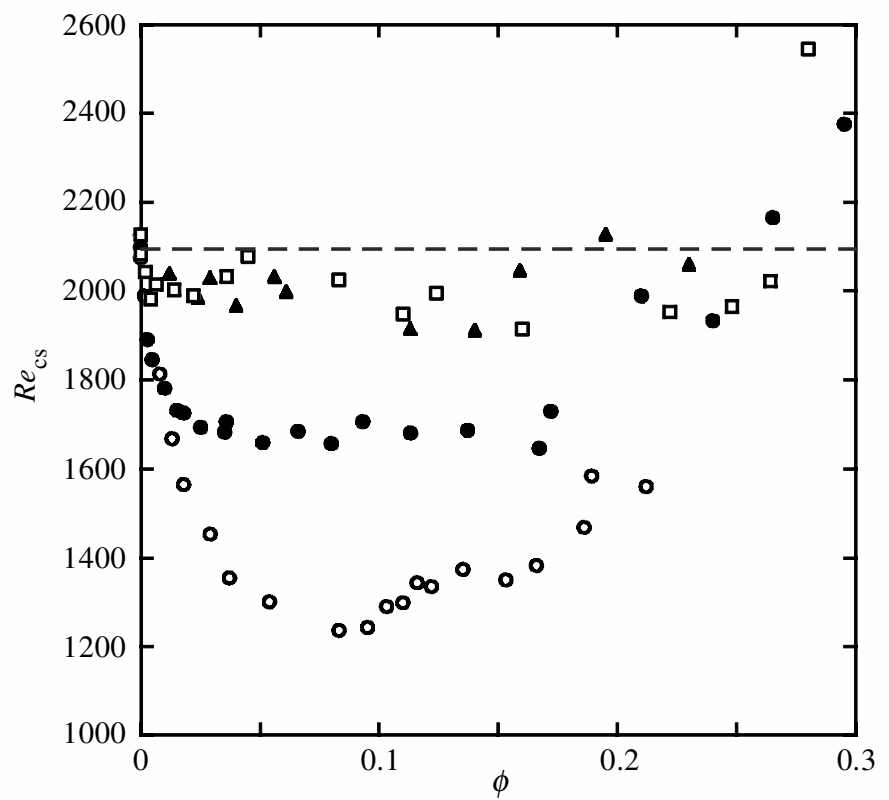

Figure 4. Critical Reynolds number of the suspension using Krieger's viscosity, $R e_{\mathrm{cs}}=R e_{\mathrm{c}} \mu_{0} / \mu_{\mathrm{e}}$, as a function of particle volume fraction, $\phi . \Delta, d=40 \mu \mathrm{m} ; \square, d=215 \mu \mathrm{m} ; \bullet, d=510 \mu \mathrm{m}$; $\circ, d=780 \mu \mathrm{m}$.

enhancement of the viscosity due to the suspended particles. Common models for the effective viscosity, $\mu_{\mathrm{e}}$, of a suspension do not depend on the size of the beads and provide a viscosity increasing with the average concentration $\phi$. A well-known example is Krieger's viscosity (Krieger 1972; Phillips et al. 1992), which expresses $\mu_{\mathrm{e}}$ as a function of $\phi$ according to

$$
\frac{\mu_{\mathrm{e}}}{\mu}=\left(1-\frac{\phi}{\phi_{\mathrm{m}}}\right)^{-1.82},
$$

where $\mu$ is the viscosity of the pure fluid and $\phi_{\mathrm{m}}=0.68$ is the close-packing (maximum) concentration for spherical particles. This empirical formula was determined for low Reynolds number but is commonly used at finite Reynolds numbers. We plot the critical Reynolds number of the suspension using this concentration-dependent viscosity, i.e. $R e_{\mathrm{cs}}=R e_{\mathrm{c}} \mu / \mu_{\mathrm{e}}$, in figure 4 . We find that for the small particles $R e_{\mathrm{cs}}$ is approximately independent of the concentration and remains close to the value for the pure fluid, $R e_{\mathrm{cs}} \approx 2100$. For $\phi>0.25$, we observe a steep increase in this threshold, suggesting the presence of an additional mechanism for dissipation beyond the viscosity enhancement observed in Stokes flow suspensions. However, we have only a limited range of concentration data, because the large flow rates required to achieve transition at the elevated effective viscosity result in a large pressure drop. The large pressure exceeds the range of the pressure gauges and also necessitates the use of the pump for $\phi>0.3$. We have chosen not to consider pump-driven flows in this work.

We now examine the dependence of the transition upon concentration for large particles. Figure 5 presents a blow-up of figure 3 for small concentrations. At first, at very low volume fractions, $R e_{\mathrm{c}}$ decreases steeply with increasing volume fraction and 


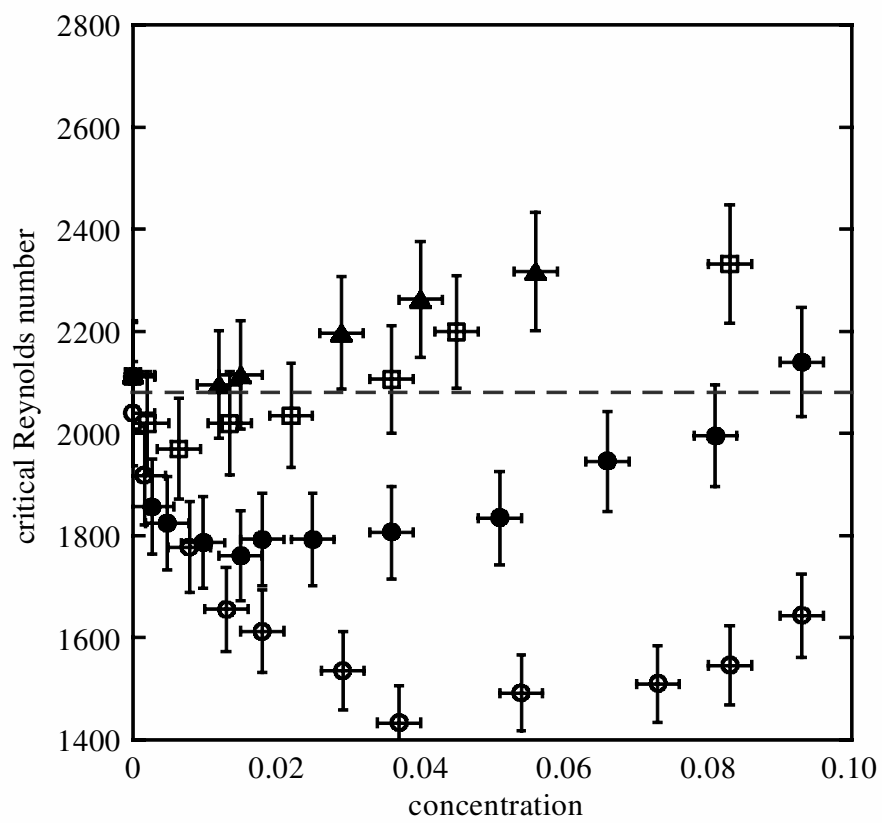

Figure 5. Critical Reynolds number, $R e_{\mathrm{c}}$, as a function of the volume fraction of the suspension for the different sets of particles (blow-up). $\boldsymbol{\Delta}, d=40 \mu \mathrm{m} ; \square, d=215 \mu \mathrm{m} ; \bullet, d=510 \mu \mathrm{m}$; $\circ, d=780 \mu \mathrm{m}$.

Table 1. Particle diameters and values of the particle Reynolds numbers for a pipe Reynolds number $R e=2100$

\begin{tabular}{lllll}
\hline$d(\mu \mathrm{m})$ & 40 & 215 & 510 & 780 \\
\hline$R e_{\mathrm{p}}$ & 0.017 & 0.5 & 2.8 & 6.5
\end{tabular}

the curves for the different particle sizes appear to collapse. It should be mentioned that a very small decrease is also observed for the $215 \mu \mathrm{m}$ particles, but this can be considered to be within error. For larger concentrations, the behaviour and in particular the minimum $R e_{\mathrm{c}}$ (when the concentration is varied) depend upon particle size. In particular, for the $780 \mu \mathrm{m}$ particles, intermittency is observed at $R e_{\mathrm{c}}=1400$ for $\phi=0.04$. This is the smallest $R e_{\mathrm{c}}$ found in this work. Similar results were also observed without the ring at the entrance, suggesting that the transition is indeed triggered by the particles. For $d=510 \mu \mathrm{m}(D / d \doteq 27.5)$, the transition is delayed for $\phi>0.10$ and for $d=780 \mu \mathrm{m}(D / d \doteq 18)$ it is delayed for $\phi>0.15$. For $\phi>0.25$, the curves seem to collapse with those for the small particles as can be seen in figure 3 . We note that the shift towards smaller $R e_{\mathrm{c}}$ occurs for particles for which the particle Reynolds number, $\operatorname{Re} e_{\mathrm{p}}=\operatorname{Re}\left(d^{2} / D^{2}\right)$, is larger than 1 , as can be seen in table 1 . One can suspect that the velocity fluctuations induced by the particles depend upon $R e_{\mathrm{p}}$ and that for large enough $R e_{\mathrm{p}}$ the transition is triggered by the noise induced by the particles.

We should also mention that large particles are known to affect the flow and enhance turbulence intensity for fully developed turbulent flows. We may assume 
that particles need to be larger than the Kolmogorov scale, $l_{K} \sim D R e^{-3 / 4}$ (ca. $50 \mu \mathrm{m}$ for the present experiment), to participate in turbulence (Landau \& Lifchitz 1994). This length-scale may give the order of magnitude of a minimum size above which particles can enhance turbulence. This is in qualitative agreement with the present results, but it should be mentioned that the flow at the threshold of transition is intermittent and turbulence is not yet fully developed.

We note that the influence of particles on the velocity profile in pipe flow is known to be a function of the particle concentration and size. In conditions of very low Reynolds number and high concentration, particles migrate towards the centre of the pipe and blunt the velocity profile (see, for example, Hampton et al. 1997), but this effect is not observed in the present experiment: the entry length $L$ of this migration is given by $L / D>1000$ for the largest particles at $\phi=0.3$ (Nott \& Brady 1994), while our tube length is less than 200 diameters (at smaller $\phi$ and for smaller particles, the entry length is larger). However, there exists a second type of migration, the so-called 'tubular pinch effect', which is inertial and causes a single particle to move to a position at a distance of $0.3 D$ from the axis (Segré \& Silberberg 1962; Han et al. 1999). This effect is, in fact, observed with the larger particles used in the present experiment. A further point is that particles introduce fluctuational velocities to the system whose form and coupling to the mean flow may vary with $R e_{\mathrm{p}}$. Unlike the case of uniform flow, the influence of $R e_{\mathrm{p}}$ on the structure of the dual wakes caused by shear flow around suspended bodies is, while under study (Zettner \& Yoda 2001; Bottin et al. 1997, 1998), not well understood. The issues raised here lead naturally to a question to be answered in the future: do the particles alter the threshold of the subcritical transition through coupling of the base flow to velocity fluctuations, or because their migration alters the base flow itself?

\section{Conclusion}

This work leads to two definite conclusions regarding the influence of suspended neutrally buoyant solids upon the transition away from laminar flow. The influence depends upon the particle size and concentration. The first conclusion is that particles can cause a delay in transition to larger $R e_{\mathrm{c}}$ : for sufficiently small particles at all $\phi$ and for larger particles above a critical volume fraction, e.g. for $D / d=18$ above $\phi=0.15$, the transition to turbulence is delayed. This behaviour becomes independent of the size of the suspended particles at $\phi>0.25$ for the range $18 \leqslant D / d \leqslant 280$ investigated here. This effect can be partly explained by the enhancement of the effective viscosity of the suspension. However, for volume fractions larger than $25 \%$, the delay in transition is found to be substantially larger than can be explained by a simple renormalization by effective viscosities. A second, and quite different, conclusion is that particles may actually induce transition at smaller $R e_{\mathrm{c}}$. For low volume fraction and above a certain critical particle size, neutrally buoyant particles alter the transition to turbulence in pipe flow to smaller Reynolds numbers. The effect increases when increasing the size of the particles.

Since this work was completed, we have examined different particle to pipe ratios in an effort to develop the scaling of the phenomena (Matas et al. 2003).

We thank P. Manneville for discussions and F. Ratouchniak for technical assistance. The donation of the $40 \mu \mathrm{m}$ particles secured by Derin Adebekun of Kodak is greatly appreciated. We also acknowledge support for this work from the Institut Français du Pétrole (French Petroleum 
Institute). This study was also undertaken under the auspices of a CNRS-NSF collaborative research project 'Flow, resuspension, and sedimentation of a suspension in a tube'. Fellowship support from the Ministère de la Recherche is gratefully acknowledged by J.-P.M.

\section{References}

Bottin, S., Dauchot, O. \& Daviaud, F. 1997 Intermittency in a locally forced plane Couette flow. Phys. Rev. Lett. 79, 4377-4380.

Bottin, S., Dauchot, O., Daviaud, F. \& Manneville, P. 1998 Experimental evidence of streamwise vortices as finite amplitude solutions in transitional plane Couette flow. Phys. Fluids 10, 2597-2607.

Crowe, C. T., Troutt, T. R. \& Chung, J. N. 1996 Numerical models for two-phase turbulent flows. A. Rev. Fluid Mech. 28, 11-43.

Darbyshire, A. G. \& Mullin, T. 1995 Transition to turbulence in constant-mass-flux pipe flow. J. Fluid Mech. 289, 83-114.

Drazin, P. G. \& Reid, W. H. 1981 Hydrodynamic stability. Cambridge University Press.

Eliahou, S., Tumin, A. \& Wygnanski, I. 1998 Laminar-turbulent transition in Poiseuille pipe flow subject to periodic perturbation emanating from the wall. J. Fluid Mech. 361, 333-349.

Hampton, R. E., Mammoli, A. A., Graham, A. L., Tetlow, N. \& Altobelli, S. A. 1997 Migration of particles undergoing pressure-driven flow in a circular conduit. J. Rheol. 41, 621-640.

Han, M., Kim, C., Kim, M. \& Lee, S. 1999 Particle migration in tube flow of suspensions. J. Rheol. 43, 1157-1173.

Krieger, I. M. 1972 Rheology of monodispersed lattices. Adv. Colloid Interface Sci. 3, 111-136.

Landau, L. \& Lifchitz, E. 1994 Mécanique des fluides, p. 191. Paris: Editions Ellipses.

Li, C., Mosyak, A. \& Hetsroni, G. 1999 Direct numerical simulation of particle-turbulence interaction. Int. J. Multiph. Flow 25, 187-200.

Matas, J.-P., Morris, J. F. \& Guazzelli, É. 2003 Transition to turbulence in particulate pipe flow. Phys. Rev. Lett. 90, 014501.

Nott, P. R. \& Brady, J. F. 1994 Pressure driven flow of suspensions: simulation and theory. J. Fluid Mech. 275, 157-199.

Phillips, R. J., Armstrong, R. C., Brown, R. A., Graham, A. L. \& Abbott, J. R. 1992 A constitutive equation for concentrated suspensions that accounts for shear-induced particle migration. Phys. Fluids A 4, 30-40.

Reynolds, O. 1883 Experimental investigation of the circumstances which determine whether the motion of water shall be direct or sinuous. Phil. Trans. R. Soc. Lond. 174, 935-982.

Segré, G. \& Silberberg, A. 1962 Behaviour of macroscopic rigid spheres in Poiseuille flow. J. Fluid Mech. 14, 136-157.

Tritton, D. J. 1988 Physical fluid dynamics. Oxford: Oxford Science Publications.

Zettner, C. M. \& Yoda, M. 2001 The circular cylinder in simple shear at moderate Reynolds numbers: an experimental study. Exp. Fluids 30, 346-353. 